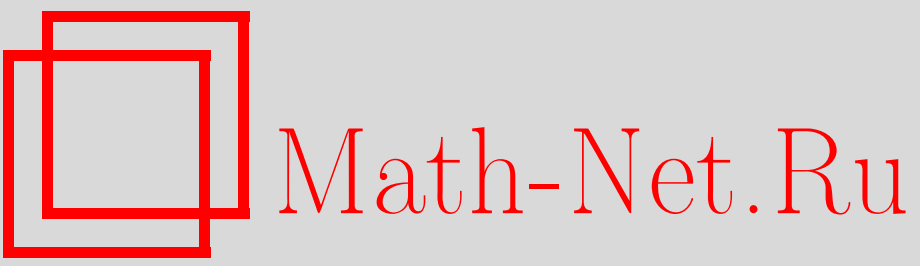

А. А. Славнов, Калибровочно-инвариантная $U(1)$-модель на некоммутативной плоскости в аксиальной калибровке, ТМФ, 2004, том 140, номер 3, 388-395

DOI: https://doi.org/10.4213/tmf105

Использование Общероссийского математического портала Math-Net.Ru подразумевает, что вы прочитали и согласны с пользовательским соглашением

http://www. mathnet.ru/rus/agreement

Параметры загрузки:

IP: 52.6 .47 .48

26 апреля 2023 г., 14:48:36 
ТЕОРЕТИЧЕСКАЯ

И МАТЕМАТИЧЕСКАЯ

ФИЗИКА

Том 140, № 3

сентябрь, 2004

(C) 2004 г.

А. А. Славнов*

\section{КАЛИБРОВОЧНО-ИНВАРИАНТНАЯ U(1)-МОДЕЛЬ НА НЕКОММУТАТИВНОЙ ПЛОСКОСТИ В АКСИАЛЬНОЙ КАЛИБРОВКЕ}

Калибровочно-инвариантная $U(1)$-модель на некоммутативной плоскости, описывающая скалярную частицу, исследована в аксиальной калибровке. Использование этой калибровки делает очевидными отсутствие неинтегрируемых инфракрасных сингулярностей и существование гладкого коммутативного предела.

Ключевые слова: некоммутативная теория поля, (UV-IR)-смешивание.

\section{1. ВВЕДЕНИЕ}

Характерной особенностью квантовых некоммутативных моделей является возникновение неинтегрируемых инфракрасных (ИK) расходимостей - (UV-IR)-смешивание. Некоторые ультрафиолетово (УФ) сходяшиеся однопетлевые диаграммы имеют сингулярности при нулевых импульсах. Это приводит к сушественной модификации закона дисперсии, которая делает физическую интерпретацию соответствуюших состояний довольно затруднительной. Более того, при рассмотрении многопетлевых диаграмм может встретиться ситуация, когда диаграмма включает в качестве подграфов однопетлевые диаграммы, имеюшие ИК-полюсы. Соответствуюшие интегралы могут содержать неинтегрируемые ИК-сингулярности. В результате в квантовых некоммутативных моделях присутствуют не только УФ-сингулярности, которые могут быть устранены с помошью подходящей процедуры перенормировки, но также ИК-сингулярности, делающие теорию несамосогласованной [1]-[10]. ИК-полюсы появляются в условно сходящихся диаграммах, для которых формальный УФ-индекс расходимости равен 1 или 2. Поэтому кажется вероятным, что такие полюсы должны отсутствовать в перенормируемых суперсимметричных моделях, в которых в коммутативном случае присутствуют только логарифмические расходимости. Действительно, явное вычисление однопетлевых диаграмм в $N=1$ суперсимметричных калибровочных моделях подтверждает эту гипотезу [11]-[13]. Если в этом случае ИК-полюсы отсутствуют и в многопетлевых диаграммах, то суперсимметричные некоммутативные модели могут быть свободны от

* Математический институт им. В. А. Стеклова РАН, Москва, Россия. E-mail:slavnov@mi.ras.ru 
трудностей, связанных с (UV-IR)-смешиванием. В настояшее время отсутствие неинтегрируемых ИК-особенностей было доказано в произвольном порядке теории возмущений для суперсимметричной модели Весса-Зумино [14] и для трехмерной суперсимметричной $U(1)$-модели [15].

Можно было бы думать, что суперсимметрия является необходимым условием существования непротиворечивых некоммутативных моделей. Однако недавно мы предложили несуперсимметричную $U(1)$-модель на пространственноподобной некоммутативной плоскости, в которой, по-видимому, отсутствуют неинтегрируемые ИК-сингулярности [16]. Доказательство, приведенное в работе [16], основывалось на сокрашении ИК-расходимостей, которому не удалось дать достаточно ясного физического объяснения.

В настоящей работе будет показано, что подходящим выбором условия калибровки можно сделать вполне очевидным отсутствие ИК-полюсов в диаграммах любого порядка. Становится также понятно, почему модели на некоммутативной пространственноподобной плоскости отличаются от других некоммутативных моделей и допускают гладкий предельный переход к коммутативному случаю.

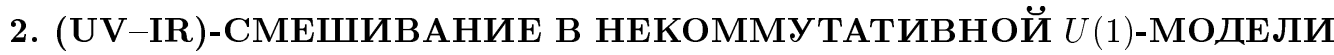

Действие некоммутативной $U(1)$-модели имеет вид

$$
\begin{aligned}
S & =\int d^{4} x\left\{-\frac{1}{4} F_{\mu \nu} F_{\mu \nu}\right\}, \\
F_{\mu \nu} & =\partial_{\mu} A_{\nu}-\partial_{\nu} A_{\mu}+i g\left[A_{\mu}, * A_{\nu}\right] .
\end{aligned}
$$

Звездочка обозначает мойаловское произведение, которое может быть записано в виде

$$
f(x) * g(x)=\left.e^{i \xi \theta_{\mu \nu} \partial_{\mu}^{x} \partial_{\nu}^{y}} f(x) g(y)\right|_{y=x},
$$

где $\theta_{\mu \nu}$ - вешественная антисимметричная матрица, а $\xi$ - параметр некоммутативности. В пределе $\xi \rightarrow 0$ действие (1) сводится к обычному максвелловскому действию для электромагнитного поля.

Калибровочное преобразование имеет вид, подобный неабелеву калибровочному преобразованию

$$
\delta A_{\mu}=\partial_{\mu} \epsilon-i g\left(A_{\mu} * \epsilon-\epsilon * A_{\mu}\right) .
$$

Отметим, что хотя для кососимметричной матрицы $\theta_{\mu \nu}$ обшего вида действие $(1)$ нелокально, в моделях с $\theta_{i 0}=0$ отсутствует нелокальность по времени и можно использовать обычный гамильтонов формализм. В дальнейшем мы предполагаем, что $\theta_{i 0}=0$ и, следовательно, можно пользоваться гамильтоновым формализмом. Мы выберем $\theta_{12}=$ $-\theta_{21}=1, \theta_{13}=\theta_{23}=0$.

В этом случае квантование проводится стандартным образом и правила Фейнмана выглядят аналогично соответствующим правилам в теории Янга-Миллса. Основным отличием является появление осциллируюших факторов в вершинах взаимодействия. 
Трехточечная вершина калибровочного поля с импульсами $k_{1}, k_{2}, k_{3}$ и индексами $\mu, \nu$, $\rho$ имеет вид

$$
2 i g \sin \left(\xi k_{1} \tilde{k}_{2}\right)\left[\left(k_{1}-k_{2}\right)_{\rho} \delta_{\mu \nu}+\left(k_{2}-k_{3}\right)_{\mu} \delta_{\nu \rho}+\left(k_{3}-k_{1}\right)_{\nu} \delta_{\mu \rho}\right]
$$

а четырехточечная вершина выглядит следующим образом:

$$
-4 g^{2}\left[\left(g^{\mu \rho} g^{\nu \sigma}-g^{\mu \sigma} g^{\nu \rho}\right) \sin \left(k_{1} \tilde{k}_{3} \xi\right) \sin \left(k_{2} \tilde{k}_{4} \xi\right)+\mathrm{sym}\right]
$$

где sym обозначает симметризацию по отношению к замене импульсов $k_{i}$ и индексов $\mu$, $\nu, \rho$. Мы пользуемся обозначением $\tilde{k}_{\mu}=\theta_{\mu \nu} k_{\nu}$.

Осциллируюшие факторы в вершинах взаимодействия подавляют часть УФ-расходимостей и в то же время приводят к появлению ИК-особенностей. В частности, поляризационный оператор калибровочного поля имеет УФ-расходящуюся часть, соответствуюшую планарным диаграммам, и сходящуюся непланарную часть, которая содержит члены, сингулярные при $p=0$. Явное вычисление дает

$$
\Pi_{\mu \nu}(p)=\frac{g^{2}}{2 \pi^{2}} \frac{\tilde{p}_{\mu} \tilde{p}_{\nu}}{\xi^{2}\left(\tilde{p}^{2}\right)^{2}}+\cdots
$$

где многоточием обозначены члены, пропорциональные $\ln \left(\xi \tilde{p}^{2}\right)$, и члены, регулярные при $p=0$.

Аналогичные сингулярности появляются в трехточечной функции, которая имеет вид

$$
\Gamma_{\mu \nu \rho}(p, q) \sim \cos (\xi p \tilde{q})\left\{\frac{\tilde{p}_{\mu} \tilde{p}_{\nu} \tilde{p}_{\rho}}{\xi\left(\tilde{p}^{2}\right)^{2}}+\operatorname{sym}\right\}+\cdots,
$$

где многоточие опять обозначает менее сингулярные члены, а sym обозначает симметризацию

$$
p \rightarrow q, \quad \mu \rightarrow \nu ; \quad p \rightarrow-(p+q), \quad \mu \rightarrow \rho .
$$

Все остальные диаграммы либо регулярны при нулевых импульсах, либо имеют логарифмические ИК-сингулярности. Полюсные ИК-сингулярности возникают в диаграммах, которые в отсутствие фазовых множителей имели бы квадратичные или линейные УФ-расходимости. Логарифмически-расходяшиеся диаграммы порождают только логарифмические расходимости, не препятствующие интегрируемости. В коммутативном случае калибровочная инвариантность обеспечивает отсутствие линейных и квадратичных расходимостей, но в некоммутативной теории они появляются. Хотя в классической теории при достаточно малом $\xi$ некоммутативность приводит к малым отклонениям от коммутативного случая, в квантовой теории некоммутативность никоим образом нельзя рассматривать как малый эффект. Даже в суперсимметричных теориях, в которых ИК-сингулярности имеют логарифмический характер, предел $\xi \rightarrow 0$ не сушествует. Единственным возможным исключением является $N=4$ теория Янга-Миллса. 
В недавней работе [16] мы предположили, что присутствие неперенормируемых расходимостей в некоммутативной $U(1)$-модели указывает на то, что данная теория неполна, и классическое действие должно быть модифицировано путем добавления новых калибровочно-инвариантных членов.

Классическое действие, предложенное в работе [16], имеет вид

$$
A=\int d^{4} x\left\{-\frac{1}{4} F_{\mu \nu} F_{\mu \nu}+\lambda(x) \theta_{\mu \nu} F_{\mu \nu}(x)\right\},
$$

где $\lambda(x)$ - множитель Лагранжа, преобразуюшийся по присоединенному представлению калибровочной группы.

Вариация действия (10) по $\lambda(x)$ приводит к новому уравнению связи

$$
\theta_{\mu \nu} F_{\mu \nu}(x)=0
$$

которое уменьшает число физических степеней свободы. Хотя действие (10) записано в терминах векторного поля $A_{\mu}$, оно описывает частицу со спином, равным нулю. Мы привели аргументы в пользу того, что в этой модели неинтегрируемые ИК-сингулярности не дают вклада в физические матричные элементы, и в пределе $\xi \rightarrow 0$ лоренц-инвариантность восстанавливается. Аргументы, приведенные в работе [16], основывались на обращении в нуль некоторых матричных элементов, что было показано прямыми вычислениями в модели на двумерной пространственноподобной некоммутативной плоскости. Нам не удалось найти очевидный механизм, ответственный за эти сокрашения. Ниже мы покажем, что отсутствие неинтегрируемых ИК-сингулярностей является прямым следствием симметрии модели, если выбрать подходящее условие калибровки.

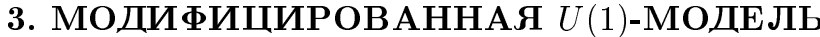 В АКСИАЛЬНОЙ КАЛИБРОВКЕ}

В отличие от работы [16], здесь мы проводим процедуру квантования в аксиальной калибровке $A_{1}=0$. В этой калибровке уравнение связи (11) сводится к условию

$$
\partial_{1} A_{2}=0
$$

где принято во внимание, что отличными от нуля элементами матришы $\theta_{\mu \nu}$ являются $\theta_{12}$ и $\theta_{21}$. Считая, что выполнены обычные асимптотические условия $A_{\mu}(x) \rightarrow 0$ при $\vec{x} \rightarrow \infty$, мы приходим к выводу, что из (12) следует, что $A_{2}=0$.

В выбранной калибровке классическое действие (10) имеет вид

$$
A=\frac{1}{2}\left(\partial_{0} A_{3}-\partial_{3} A_{0}+i g\left[A_{0}, * A_{3}\right]\right)^{2}+\frac{1}{2} \sum_{i=1,2}\left(\partial_{i} A_{0}\right)^{2}-\frac{1}{2} \sum_{i=1,2}\left(\partial_{i} A_{3}\right)^{2} .
$$

Это действие инвариантно относительно врашений в плоскости $(0,3)$. Вводя канонические импульсы

$$
p_{3}=\frac{\partial L}{\partial \dot{A}_{3}}=F_{03}
$$


можно переписать действие в виде

$$
A=\int\left\{p_{3} \dot{A}_{3}-\frac{1}{2} p_{3}^{2}+A_{0} D_{3} p_{3}+\frac{1}{2} \sum_{i=1,2}\left(\partial_{i} A_{0}\right)^{2}-\frac{1}{2} \sum_{i=1,2}\left(\partial_{i} A_{3}\right)^{2}\right\} d x
$$

где $D_{3}$ обозначает ковариантную производную. Переменную $A_{0}$ можно исключить, решив не содержашее производных по времени уравнение

$$
A_{0}=\nabla^{-2} D_{3} p_{3}, \quad \nabla^{2}=\partial_{1}^{2}+\partial_{2}^{2}
$$

Подставляя это решение в действие (15), получаем

$$
A=\int\left\{p_{3} \dot{A}_{3}-\frac{1}{2} p_{3}^{2}-\frac{1}{2} D_{3} p_{3} \nabla^{-2} D_{3} p_{3}-\frac{1}{2} \sum_{i=1,2}\left(\partial_{i} A_{3}\right)^{2}\right\} d x
$$

Это действие описывает скалярную частищу с гамильтонианом

$$
H=\frac{p_{3}^{2}}{2}+\frac{1}{2} D_{3} p_{3} \nabla^{-2} D_{3} p_{3}-\frac{1}{2} \sum_{i=1,2}\left(\partial_{i} A_{3}\right)^{2},
$$

и к нему можно применить обьчную процедуру канонического квантования.

Действие (10) не инвариантно относительно преобразований Лоренца, однако, как будет показано, в классическом коммутативном пределе $\xi \rightarrow 0$ инвариантность теории восстанавливается.

В самом деле, в коммутативном пределе действие принимает вид

$$
A=\int\left\{p_{3} \dot{A}_{3}-\frac{1}{2} p_{3}^{2}-\frac{1}{2} \partial_{3} p_{3} \nabla^{-2} \partial_{3} p_{3}-\frac{1}{2} \sum_{i=1,2}\left(\partial_{i} A_{3}\right)^{2}\right\} d x
$$

Переопределяя переменные

$$
\begin{aligned}
& \pi(k)=\left[1+k_{3}^{2}\left(k_{1}^{2}+k_{2}^{2}\right)^{-1}\right]^{1 / 2} \tilde{p}_{3}(k), \\
& \phi(k)=\left[1+k_{3}^{2}\left(k_{1}^{2}+k_{2}^{2}\right)^{-1}\right]^{-1 / 2} \tilde{A}_{3}(k),
\end{aligned}
$$

его легко свести к стандартному лоренц-инвариантному действию, описывающему свободную скалярную частицу:

$$
A=\int\left\{\pi \dot{\phi}-\frac{1}{2} \pi^{2}-\frac{1}{2} \sum_{i=1}^{3}\left(\partial_{i} \phi\right)^{2}\right\} d x
$$

Однако квантовые поправки могут нарушить этот гладкий переход, как это случается в обычной некоммутативной $U(1)$-модели. Ниже мы покажем, что в нашем случае гладкий переход к модели свободной скалярной частицы существует и в квантовом случае. Все квантовые поправки исчезают в пределе $\xi \rightarrow 0$. 
Как обычно, матрицу рассеяния можно представить в виде континуального интеграла от $e^{i A}$, где $A$ определяется уравнением (17). Соответствуюшее равенство удобно записать, вводя интегрирование по $A_{0}$ :

$$
\begin{aligned}
S= & \int \exp \left\{i \int \left[p_{3} \dot{A}_{3}-\frac{1}{2} p_{3}^{2}+A_{0} D_{3} p_{3}+\right.\right. \\
& \left.\left.+\frac{1}{2} \sum_{i=1,2}\left(\partial_{i} A_{0}\right)^{2}-\frac{1}{2} \sum_{i=1,2}\left(\partial_{i} A_{3}\right)^{2}\right] d x\right\} d A_{3} d A_{0} d p
\end{aligned}
$$

где подразумеваются обычные граничные условия на $A_{3}$.

Выполняя гауссово интегрирование по $p_{3}$, получаем

$$
S=\int \exp \left\{i \int\left[\frac{1}{2} F_{03}^{2}+\frac{1}{2} \sum_{i=1,2}\left(\partial_{i} A_{0}\right)^{2}-\frac{1}{2} \sum_{i=1,2}\left(\partial_{i} A_{3}\right)^{2}\right] d x\right\} d A_{3} d A_{0}
$$

Пропагатор получается обрашением квадратичной формы, входящей в свободную часть действия (24), и имеет вид

$$
D_{\mu \nu}(k)=\frac{1}{k^{2}+i \epsilon}\left\{g^{\mu \nu}-\frac{k_{\mu} k_{\nu}}{k_{1}^{2}+k_{2}^{2}}\right\}, \quad \mu, \nu=0,3 .
$$

Все остальные элементы $D_{\mu \nu}$ равны нулю. Поэтому пропагатор (25), очевидно, удовлетворяет условию поперечности вида

$$
\tilde{k}_{\mu} D_{\mu \nu}(k)=0 \text {. }
$$

В силу калибровочной инвариантности действия (10) функции Грина удовлетворяют обобщенным тождествам Уорда

$$
\begin{aligned}
& \int \exp \left\{i A+\int\left[\frac{1}{2 \alpha}\left(A_{1}\right)^{2}+J_{\mu} A_{\mu}\right] d x\right\} \times \\
& \quad \times\left(\frac{1}{\alpha} \partial_{1} A_{1}(x)-\partial_{\mu} J_{\mu}(x)+i g\left[A_{\mu}(x), * J_{\mu}(x)\right]\right) d A_{\mu}=0 .
\end{aligned}
$$

Для двухточечной функции Грина имеем

$$
-\frac{i}{\alpha}\left\langle\partial_{1} A_{1}(x) A_{\nu}(y)\right\rangle=\partial_{\nu} \delta(x-y)
$$

Фурье-образ поляризационного оператора удовлетворяет условию поперечности

$$
p_{\mu} \Pi_{\mu \nu}(p)=0 \text {. }
$$

Рассмотрим поляризационный оператор более детально. Легко показать, что сингулярные полюсные члены в этой функции зависят только от $\tilde{p}$. В самом деле, поляризационный оператор имеет вид

$$
\Pi_{\mu \nu}(p)=\int \prod_{i} d k_{i} \sin \left(k_{1} \tilde{p} \xi\right) \sin \left(k_{n} \tilde{p} \xi\right)\left[f_{\mu \nu}\left(k_{1}, \ldots, k_{n}, p_{0}, p_{3}, \tilde{p}\right)\right],
$$


где мы обозначили импульсы, входящие во внешние вершины, как $k_{1}$ и $k_{n}$. Подынтегральное выражение в этой формуле представляет собой рациональную функцию размерности $p^{-4 n+2}$, умноженную на произведение тригонометрических функций. Оператор $\Pi_{\mu \nu}(p)$ можно представить в виде

$$
\begin{aligned}
\Pi_{\mu \nu}(p)= & \int\left[f_{\mu \nu}\left(k_{1}, \ldots, k_{n}, p_{0}, p_{3}, \tilde{p}\right)-f_{\mu \nu}\left(k_{1}, \ldots, k_{n}, 0,0, \tilde{p}\right)\right] \times \\
& \times \sin \left(k_{1} \tilde{p} \xi\right) \sin \left(k_{n} \tilde{p} \xi\right) \prod_{i} d k_{i}+ \\
& +\int f_{\mu \nu}\left(k_{1}, \ldots, k_{n}, 0,0, \tilde{p}\right) \sin \left(k_{1} \tilde{p} \xi\right) \sin \left(k_{n} \tilde{p} \xi\right) \prod_{i} d k_{i}
\end{aligned}
$$

Первый член не может породить ИК-полюс второго порядка, поскольку интеграл расходится по индексу не более чем линейно. В действительности по соображениям симметрии расходимость логарифмическая. Следовательно, полюсные ИК-сингулярности могут присутствовать лишь во втором члене, зависящем только от $\tilde{p}$.

Единственная возможная структура, зависящая только от $\tilde{p}$ и совместимая с тождеством (27), имеет вид

$$
\Pi_{\mu \nu}^{\mathrm{pole}}(p)=\tilde{p}_{\mu} \tilde{p}_{\nu} \Pi(\tilde{p})
$$

Напомним, что свободный пропагатор поля $A_{\mu}$, определяемый формулой $(25)$, удовлетворяет условию поперечности (26). Поэтому вклад ИК-полюсов поляризационного оператора $\Pi_{\mu \nu}(p)$ в любую диаграмму Фейнмана обращается в нуль. Могут присутствовать лишш логарифмические ИК-сингулярности $\sim \ln \left(\tilde{p}^{2} \xi\right)$. Эти особенности интегрируемы и не приводят к противоречивости теории.

Отсутствие ИК-полюсов в рассматриваемой калибровке легко понять. Ненулевые элементы пропагатора $D_{\mu \nu}$ отвечают $\mu, \nu=0,3$. В то же время ИК-полюсная часть поляризационного оператора $\Pi_{\mu \nu}$ зависит только от $\tilde{p}_{\mu}$ и, следовательно, пропорциональна $p_{1}$ или $p_{2}$. Очевидно, что

$$
D_{\mu \nu} \Pi_{\nu \alpha}^{\text {pole }}=0
$$

Аналогичные аргументы применимы и к трехточечной функции. По тем же причинам ее ИК-сингулярная часть зависит только от $\tilde{p}, \tilde{q}$, и, следовательно,

$$
D_{\mu \alpha}(p) \Gamma_{\alpha \nu \rho}^{\mathrm{pole}}(p, q)=D_{\nu \alpha}(q) \Gamma_{\mu \alpha \rho}^{\mathrm{pole}}(p, q)=D_{\rho \alpha}(p+q) \Gamma_{\mu \nu \alpha}^{\mathrm{pole}}(p, q)=0
$$

Как и в случае поляризационного оператора, ненулевой вклад могут давать только логарифмические ИК-сингулярности $\Gamma_{\mu \nu \rho}(p, q)$.

Все остальные диаграммы по индексу расходятся не более чем логарифмически и, следовательно, не могут порождать полюсных ИК-сингулярностей.

Итак, мы доказали, что некоммутативная $U(1)$-модель, описываемая действием (10), не порождает неинтегрируемых ИК-сингулярностей и позволяет вычислять радиационные поправки произвольного порядка. Заметим, что в коммутативном пределе $\xi \rightarrow 0$ все вершины взаимодействия исчезают как $\xi$. Принимая во внимание, что возможные 
сингулярности радиационных поправок по $\xi$ пропорциональны $\ln \xi$, мы делаем вывод, что в квантовой версии нашей модели предел $\xi \rightarrow 0$ существует и описывает свободную скалярную частицу.

Наши выводы не меняются, если $A_{\mu}$ взаимодействует с полем спина $1 / 2$ в присоединенном представлении. В модели по-прежнему отсутствуют полюсные ИК-сингулярности, и в пределе $\xi \rightarrow 0$ восстанавливается лоренц-инвариантность. Если взаимодействие включает также спинорные поля в фундаментальном представлении, то ИК-полюсные сингулярности отсутствуют, однако лоренц-инвариантный предел при $\xi \rightarrow 0$ может отсутствовать, что имеет место в суперсимметричных моделях.

\section{4. ЗАКЛЮЧЕНИЕ}

В данной работе была рассмотрена самосогласованная калибровочно-инвариантная $U(1)$-модель на некоммутативной плоскости $\left(x_{1}, x_{2}\right)$ в аксиальной калибровке $A_{1}=0$. В этой калибровке две компоненты калибровочного поля обрашаются в нуль, и отсутствие ИК-полюсных сингулярностей в произвольном порядке теории возмущений, а также существование гладкого коммутативного предела становятся очевидными.

Благодарности. Работа была частично поддержана Российским фондом фундаментальных исследований (грант № 02-01-00126), Президентской программой поддержки ведущих научных школ (грант № НШ 2052.2003.1) и программой РАН “Теоретические проблемы математики".

\section{Список литературы}

[1] T. Filk. Phys. Lett. B. 1996. V. 376. P. 53.

[2] T. Krajewsky, R. Wulkenhaar. Int. J. Mod. Phys. A. 2000. V. 15. P. 1011.

[3] S. Minwalla, M. Van Raamsdonk, N. Seiberg. JHEP. 1999. V. 9906. P. 007.

[4] I. Ya. Aref'eva, D. M. Belov, A.S. Koshelev. Phys. Lett. B. 2000. V. 476. P. 431.

[5] H. Grosse, T. Krajewski, R. Wulkenhaar. Renormalization of noncommutative Yang-Mills theories: A simple example. hep-th/0001182.

[6] C. P. Martin, D. Sanchez-Ruiz. Phys. Rev. Lett. 1999. V. 83. P. 476.

[7] M. Hayakawa. Phys. Lett. B. 2000. V. 478. P. 394.

[8] A. Matusis, L. Susskind, N. Tombas. JHEP. 2000. V. 0012. P. 002.

[9] I. Ya. Aref'eva, D. M. Belov, A.S. Koshelev, O. A. Rytchkov. Nucl. Phys. B. 1993. V. 406. P. 90.

[10] I. Chepelev, R. Roiban. JHEP. 2001. V. 0103. P. 001

[11] M. M. Sheikh-Jabbari. JHEP. 1999. V. 9905. P. 015.

[12] D. Zanon. Phys. Lett. B. 2001. V. 502. P. 265.

[13] I. Jack, D. R. T. Jones. New J. Phys. 2001. V. 31. P. 19.

[14] H. O. Girotti, M. Gomes, V. O. Rivelles, A. J. da Silva. Nucl. Phys. B. 2000. V. 587. P. 299.

[15] A. F. Ferrari, H. O. Girotti, M. Gomes et al. Phys. Lett. B. 2003. V. 577. P. 83.

[16] A. A. Slavnov. Phys. Lett. B. 2003. V. 565. P. 246. 